\title{
Vessel Wall Enhancement and Focal Cerebral Arteriopathy in a Pediatric Patient with Acute Infarct and COVID-19 Infection
}

\author{
(D)E. Gulko, (D) P. Overby, (D) Ali, DH. Mehta, (D)F. Al-Mufti, and DW. Gomes
}

\section{ABSTRACT}

SUMMARY: Herein, we report the findings of intracranial arterial wall enhancement, consistent with focal cerebral arteriopathyinflammatory type, in a child presenting with acute infarct in the setting of coronavirus disease 2019 (COVID-19) infection. To our knowledge, this report provides the first description of vessel wall imaging findings in COVID-19-associated acute stroke.

ABBREVIATIONS: COVID-19 = coronavirus disease 2019; FCA = focal cerebral arteriopathy; SARS-CoV-2 = Severe Acute Respiratory Syndrome coronavirus 2; $\mathrm{VWI}=$ vessel wall imaging

$\mathbf{S}$ evere Acute Respiratory Syndrome coronavirus 2 (SARS$\mathrm{CoV}-2$ ) has resulted in the world-wide pandemic of coronavirus disease 2019 (COVID-19) illnesses, including Severe Acute Respiratory Syndrome and a multitude of neurologic manifestations. ${ }^{1}$ There is emerging evidence for the role of the cerebrovascular system in neurologic manifestations of COVID-19 infection, and patients with COVID-19 infection may be at greater risk for thromboembolic disease. ${ }^{2}$ Recent studies have demonstrated that patients can develop intracranial hemorrhages, acute strokes, ${ }^{3}$ and large-vessel arterial occlusions. ${ }^{4}$ The pathomechanisms that underlie COVID-19-associated cerebrovascular disease remain unclear. While growing numbers of case reports and studies have highlighted COVID-19-associated neurologic disease in adults, there are few reports of COVID-19associated neurologic disease in children. A recent case report demonstrated stenosis of the left middle cerebral artery, which was attributed to focal cerebral arteriopathy (FCA), in a pediatric patient with COVID-19 and acute stroke. ${ }^{5}$ Herein, we describe the second documented case of COVID-19-associated FCA and acute stroke in a pediatric patient. Additionally, we provide the presumptive first description of MR imaging vessel wall imaging (VWI) findings in a patient with COVID-19-related stroke.

A 13-year-old right-handed girl presented with fluctuatingbut-persistent headache, speech difficulty, and right upper and

Received June 19, 2020; accepted after revision July 16.

From the Department of Radiology, Division of Neuroradiology (E.G., S.A., H.M., W.G.), Department of Pediatrics (P.O.), and Department of Neurology (F.A.-M.), Westchester Medical Center, Valhalla, New York.

Please address correspondence to Edwin Gulko, MD, Division of Neuroradiology, Department of Radiology, Westchester Medical Center, 100 Woods Rd, Vahalla

New York, 10595; e-mail: Edwin.Gulko@wmchealth.org

- Indicates open access to non-subscribers at www.ajnr.org

http://dx.doi.org/10.3174/ajnr.A6778 lower extremity weakness for 4 days. Two months before presentation, she and other family members experienced fever, myalgias, and anosmia, which subsequently resolved. One month before admission, she and other family members tested positive for SARS-CoV-2 qualitative antibodies. Antibody testing for the patient was performed with the ADVIA Centaur SARS-CoV-2 Total Assay (Siemens). The child had no other medical history. There was no family history of thrombophilia.

The initial exam was normal for temperature, blood pressure, and heart rate. Her speech was dysfluent with word-finding difficulties. She had mild-to-moderate extensor weakness in the right arm and leg. Initial head CT showed a left frontal hypodensity (not shown) concerning for ischemic infarct. Consequently, the patient was transferred to our tertiary referral center. At our facility, results of testing for SARS-CoV-2 RNA by real-time reverse transcription polymerase chain reaction with a nasopharyngeal swab specimen were positive.

MR imaging of the brain demonstrated small regions of restricted diffusion and FLAIR hyperintensity, with the left frontal, parietal, and temporal lobes consistent with acute-subacute infarcts in the left middle cerebral artery vascular territory (Fig 1). Noncontrast MRA of the head was performed with a time-of-flight sequence. Vessel wall imaging was performed with and without intravenous gadolinium contrast with $3 \mathrm{D}$ volume isotropic turbo spin-echo acquisition T1 and black-blood sequences on a $3 \mathrm{~T}$ MR imaging instrument. MRA of the head demonstrated focal moderate stenosis within the M1 segment of the left middle cerebral artery (Fig 1). Vessel wall imaging targeted to the left middle cerebral artery M1 segment demonstrated wall thickening and marked, concentric contrast enhancement (Fig 2) at the site of stenosis. These imaging features, in conjunction with the clinical presentation, were consistent with focal cerebral arteriopathy of childhoodinflammatory type. ${ }^{6}$ 


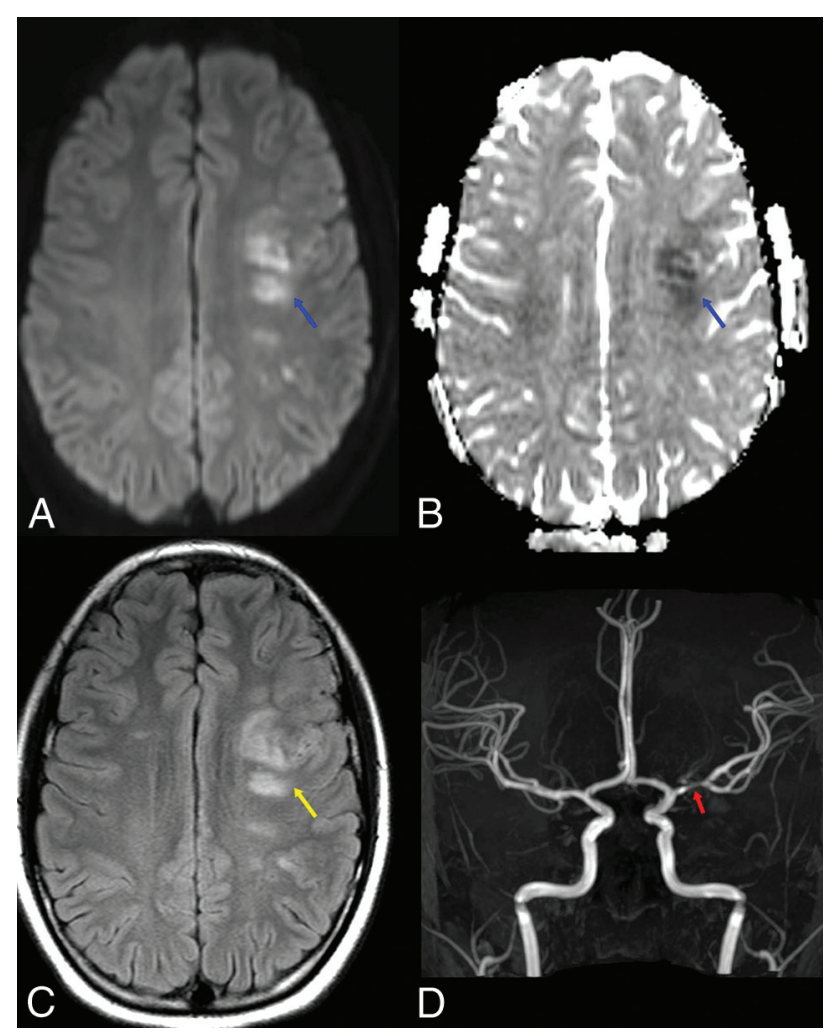

FIG 1. COVID-19-associated left MCA vascular territory acute infarcts in a pediatric patient. Axial diffusion (A), ADC map (B), and FLAIR $(C)$ images demonstrate foci of restricted diffusion (blue arrows) and cytotoxic edema (yellow arrow) within the left middle cerebral artery vascular territory, consistent with acute infarcts. Anterior projection from a TOF-MRA of the head $(D)$ demonstrates a focal segment of moderate stenosis within the left M1 middle cerebral artery (red arrow).
Additional work-up for the patient was unrevealing. The echocardiogram findings were within normal limits. The lumbar puncture result was within normal limits. Multiple viral polymerase chain reaction tests, including herpes simplex virus and varicella zoster virus, were negative from the spinal fluid, as were bacterial cultures. Thrombophilia evaluation had normal findings. Other inflammatory markers, typically elevated in COVID19-associated pediatric multisystem inflammatory syndrome, were normal. Clinically, the patient improved, and the neurologic examination was unremarkable at the time of discharge. A follow-up MR imaging examination has not yet been performed at the completion of this article.

Large-vessel cerebral arteriopathy is the most common cause of arterial ischemic stroke in a previously healthy child. ${ }^{7}$ The Vascular Effects of Infection in Pediatric Stroke study ${ }^{6}$ found that the most common arteriopathies in children presenting with acute arterial ischemic stroke included Moyamoya disease, arterial dissection, and FCA-inflammatory type. An updated definition of FCA by Wintermark et $\mathrm{al}^{6}$ includes unifocal and unilateral stenosis/irregularity of the distal internal carotid artery and/or its proximal branches. FCA-inflammatory type describes FCA that is presumed to be inflammatory (a focal vasculitis) and can be diagnosed by marked concentric vessel wall enhancement on VWI. ${ }^{6}$ FCA, dissection type, in contrast, refers to intracranial arterial dissection, typically associated with a history of trauma.

VWI is capable of distinguishing among disease entities involving the intracranial arterial system. ${ }^{8} \mathrm{CNS}$ vasculitis typically demonstrates vessel wall thickening on VWI, with homogeneous and concentric contrast enhancement, ${ }^{8}$ and infectious pathogens such as varicella zoster have been shown to cause concentric vessel wall enhancement. ${ }^{9}$ Other forms of arteriopathy can also result in vessel wall enhancement, including arterial dissection,

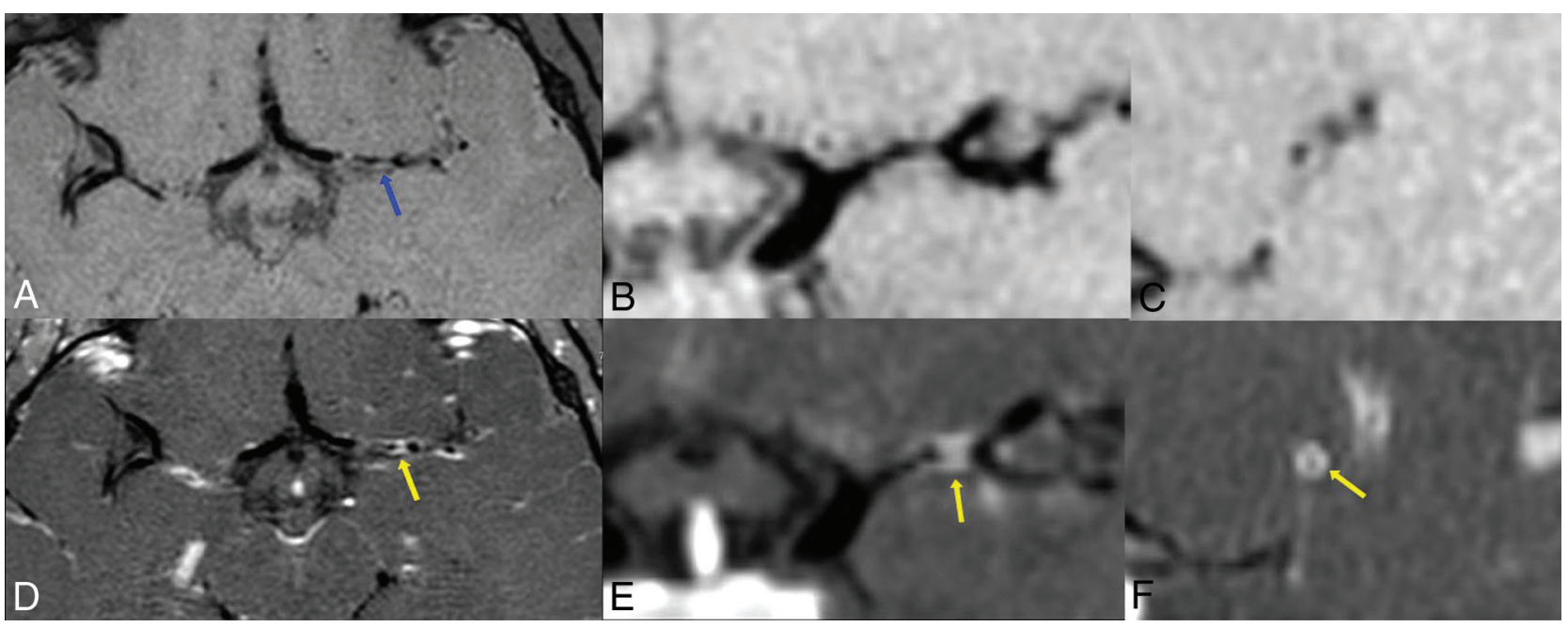

FIG 2. Vessel wall enhancement within the M1 left MCA. Vessel wall imaging targeted to the left middle cerebral artery with axial pre- $(A)$ and postcontrast (D) 3D T1 volume isotropic turbo spin-echo acquisition sequences, with reformatted coronal images; $B$ and $E$, respectively). Pre- (C) and postcontrast $(F)$ reformatted sagittal images en face to the M1 left middle cerebral artery at the level of the blue and yellow arrows, respectively. There is wall thickening (blue arrow) and marked concentric contrast enhancement of the M1 left middle cerebral artery at the segment of stenosis (yellow arrows). The imaging findings, in conjunction with the clinical history, were consistent with FCA-inflammatory type. 
cardioembolism, and drug-induced vasospasm. ${ }^{10,11}$ However, in our patient, no clinical or laboratory findings were present to support these other possibilities.

Existing evidence in the adult population supports thromboembolism as a common cause of stroke in patients with COVID $-19,{ }^{2}$ possibly secondary to "cytokine storms," which can lead to vascular endothelial damage. ${ }^{12,13}$ It is currently unknown whether the pathophysiology of COVID-19-associated acute infarcts in the pediatric population is similar to that described in adults. A recent report by Mirzaee et $\mathrm{al}^{5}$ described a case of presumed focal cerebral arteriopathy in a pediatric patient with COVID-19, but VWI was not performed. We similarly observed evidence of focal cerebral arteriopathy on MRA but were able to corroborate the diagnosis of FCA-inflammatory type with VWI. Taken together, our results and those of Mirzaee et al suggest FCA as a mechanism of SARS-CoV-2-related acute ischemic stroke in children. It is currently unclear whether FCA is also a significant cause of ischemic infarction in adults with COVID-19.

To our knowledge, this report provides the first description of vessel wall imaging findings in a patient with COVID-19 with acute ischemic stroke. We believe that VWI may facilitate the specific diagnosis of focal cerebral arteriopathy in children (and perhaps adults) with COVID-19. Of note, steroid therapy may improve the outcome in focal cerebral arteriopathy; ${ }^{14}$ recognition of focal cerebral arteriopathy on imaging may, therefore, contribute to the choice of a therapeutic regimen in COVID-19-related infarction. We, therefore, suggest that clinicians and neuroradiologists consider using vessel wall imaging to aid in the evaluation of patients with COVID-19 and acute stroke.

Disclosures: Philip Overby_UNRELATED: Expert Testimony: legal medical reviews. Fawaz Al-Mufti-UNRELATED: Employment: Westchester Medical Center at New York Medical College.

\section{REFERENCES}

1. Helms J, Kremer S, Merdji H, et al. Neurologic features in severe SARS-CoV-2 infection. $N$ Engl J Med 2020;382:2268-70 CrossRef Medline
2. Bikdeli B, Madhavan MV, Jimenez D, et al; the IUA, Supported by the ESC Working Group on Pulmonary Circulation and Right Ventricular Function. COVID-19 and thrombotic or thromboembolic disease: implications for prevention, antithrombotic therapy, and follow-up. J Am Coll Cardiol 2020;75:2950-73 CrossRef Medline

3. Radmanesh A, Raz E, Zan E, et al. Brain imaging utilization and findings in COVID-19: a single academic center experience in the epicenter of disease in the United States. AJNR Am J Neuroradiol 2020;41:1179-83 CrossRef Medline

4. Oxley TJ, Mocco J, Majidi S, et al. Large-vessel stroke as a presenting feature of Covid-19 in the young. N Engl J Med 2020;382:e60 CrossRef Medline

5. Mirzaee SM, Gonçalves FG, Mohammadifard M, et al. Focal cerebral arteriopathy in a COVID-19 pediatric patient. Radiology 2020 June 2. [Epub ahead of print] CrossRef Medline

6. Wintermark M, Hills NK, DeVeber GA, et al; the VIPS Investigators. Clinical and imaging characteristics of arteriopathy subtypes in children with arterial ischemic stroke: results of the VIPS study. AJNR Am J Neuroradiol 2017;38:2172-79 CrossRef Medline

7. Fullerton HJ, Elkind MS, Barkovich AJ, et al. The vascular effects of infection in pediatric stroke (VIPS) study. J Child Neurol 2011;26:1101-10 CrossRef Medline

8. Mandell DM, Mossa-Basha M, Qiao Y, et al; Vessel Wall Imaging Study Group of the American Society of Neuroradiology. Intracranial vessel wall MRI: principles and expert consensus recommendations of the American Society of Neuroradiology. AJNR Am J Neuroradiol 2017;38:218-29 CrossRef Medline

9. Cheng-Ching E, Jones S, Hui FK, et al. High-resolution MRI vessel wall imaging in varicella zoster virus vasculopathy. J Neurol Sci 2015;351:168-73 CrossRef Medline

10. Stence NV, Pabst LL, Hollatz AL, et al. Predicting progression of intracranial arteriopathies in childhood stroke with vessel wall imaging. Stroke 2017;48:2274-77 CrossRef Medline

11. Dlamini N, Yau I, Muthusami P, et al. Arterial wall imaging in pediatric stroke. Stroke 2018;49:891-98 CrossRef Medline

12. Ye Q, Wang B, Mao J. The pathogenesis and treatment of the “cytokine storm" in COVID-19. J Infect 2020;80:607-13 CrossRef Medline

13. Mehta P, McAuley DF, Brown M, et al; HLH Across Speciality Collaboration, UK. COVID-19: consider cytokine storm syndromes and immunosuppression. Lancet 2020;395:1033-34 CrossRef Medline

14. Steinlin M, Bigi S, Stojanovski B, et al; Swiss NeuroPediatric Stroke Registry. Focal cerebral arteriopathy: do steroids improve outcome? Stroke 2017;48:2375-82 CrossRef Medline 